\section{Expresión y amplificación del gen HER2 en el cáncer gástrico avanzado}

\author{
IVÁN ROA ${ }^{1}$, JEANNIE SLATER ${ }^{2}$, DANIEL CARVAJAL ${ }^{3}$, \\ KURT SCHALPER ${ }^{3}$, GONZALO DE TORO ${ }^{4}$, RAÚL ARES ${ }^{5}$, \\ ANAKAREN GAME ${ }^{6, a}$, JORGE LEÓN ${ }^{7}$, XABIER DE ARETXABALA ${ }^{7}$
}

\section{HER2 gene amplification and overexpression in advanced gastric cancer}

Background: Overexpression/amplification of the HER2 gene in advanced gastric cancer is a predictor of response to adjuvant therapy with monoclonal antibodies. Aim: To determine the frequency of HER2 gene overexpression and amplification in advanced gastric cancer. Material and Methods: One hundred nine advanced gastric cancer biopsy specimens, from 76 men and 33 women aged $67 \pm 14$ and 62 \pm 12 years respectively, were selected. Three histological patterns (diffuse, intestinal and mixed) were recognized. Automated immunohistochemistry was performed with monoclonal c-erbB-2 (NCL-356) Novocastra. Fluorescent in situ hybridization (FISH) for HER2 was performed in positive cases. Results: In 39\% of cases, immunohistochemical staining was negative. It was $1+, 2+$ and $3+$ positive in 15 , 36 and $11 \%$ of cases, respectively. It was positive in $16 \%$ and $3 \%$ of intestinal type and mixed carcinomas, respectively. It was negative in all diffuse carcinomas. FISH was performed in $39(2+)$ cases and in $11(3+)$ cases. The gene amplification was positive in two $(2+)$ and $11(3+)$ cases $(11.9 \%)$. The overall concordance between immunohistochemical staining and in situ hybridization was 85\%. Conclusions: In advanced gastric cancer, HER2 gene overexpression or amplification was observed in $11 \%$ and $12 \%$ of cases, respectively.

(Rev Med Chile 2013; 141: 1411-1419)

Key words: Gene amplification; HER2, protein, human; in situ hibridization, fluorescence; Immunohistochemistry.

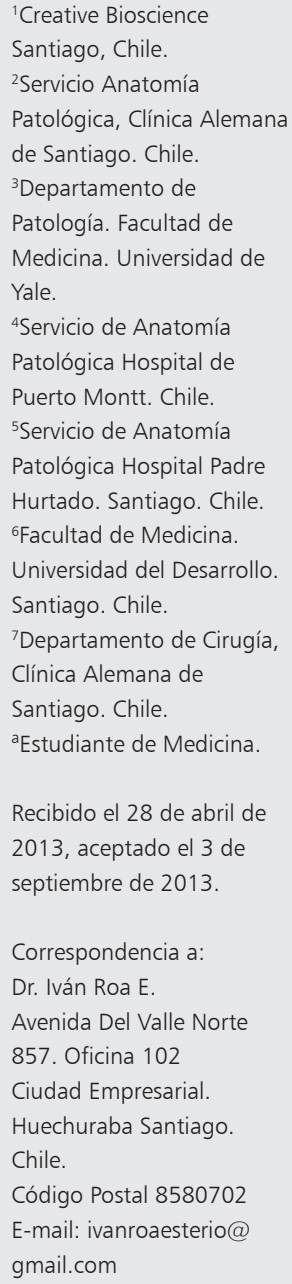

E l cáncer gástrico es la segunda causa más frecuente de muerte por cáncer en todo el mundo y representa alrededor de $8 \%$ del total de casos de cáncer y $10 \%$ de las muertes por todos los tipos de cáncer ${ }^{1-3}$, y sobre $70 \%$ ocurren en los países en desarrollo ${ }^{2,3}$. No obstante, de que su incidencia ha ido lentamente disminuyendo desde hace décadas, su pronóstico continúa siendo pobre ${ }^{4,5}$. En Chile representa un importante problema de salud pública, siendo la mayor parte de los casos diagnosticados en estadios avanzados y con pocas expectativas terapéuticas ${ }^{6,7}$. En los últimos años el tratamiento quirúrgico asociado con quimio y radioterapia ha mejorado las expectativas de vida de estos enfermos, lográndose sobrevidas sobre $50 \%$ en las formas localizadas distales de la enfermedad, en cambio, en las formas avanzadas y proximales la sobrevida a 5 años no va más allá de $10-20 \%{ }^{8-11}$.

En el cáncer gástrico avanzado se ha incorporado al tratamiento la quimioterapia estándar y la terapia con anticuerpos monoclonales como el trastuzumab en aquellos casos que presentan sobreexpresión o amplificación del gen HER $2^{12-14}$. Esto ha permitido abrir expectativas con terapias dirigidas a blancos moleculares tal y como se está empleando en otros cánceres como el cáncer de mama, colon y pulmón ${ }^{15,16}$.

Entre los cánceres gástricos de origen epitelial, se reconocen dos grandes grupos histológicos: los 
de tipo intestinal (tumores mejores diferenciados) y los difusos (con menor diferenciación), como lo señalado en la original clasificación de Lauren en $1965^{17,18}$. Ambos tipos histológicos presentan características epidemiológicas, clínicas y morfológicas diferentes, lo que refleja una patogenia y alteraciones genético-moleculares significativamente distintas ${ }^{19-21}$. Entre las principales diferencias que se observan entre los adenocarcinomas de tipo difuso e intestinal, está la pérdida de la expresión del gen de la E-caderina, cuya proteína se localiza en la superficie celular como una molécula de adhesión celular esencial para la mantención y organización de los tejidos epiteliales ${ }^{22-24}$.

El gen HER2 es un proto-oncogén localizado en el cromosoma 17 que pertenece a la familia HER que comprende a cuatro receptores diferentes $^{25}$. El gen HER2 también llamado: ErbB-2, c-erbB2 o Her2/neu, codifica una proteína de transmembrana de tipo tirosina-quinasa de 185$\mathrm{kDa}$. Estos receptores cooperan en la regulación de diferentes procesos, tales como la proliferación, la diferenciación, y la supervivencia celular. HER2 actúa como un oncogén y su amplificación se traduce principalmente como una sobreexpresión de la proteína. Esta sobreexpresión de HER2 se ha relacionado con una mayor agresividad y peor pronóstico, al facilitar la proliferación y migración de las células tumorales en algunos tumores malignos $^{26,27}$. Aproximadamente, $25 \%$ a $30 \%$ de los cánceres de mama sobreexpresan HER2, siendo un factor predictivo en la respuesta a la terapia con el anticuerpo monoclonal trastuzumab ${ }^{28,29} \mathrm{u}$ otros inhibidores del receptor como el lapatinib ${ }^{30}$. En el cáncer gástrico la sobreexpresión de HER2 se ha descrito entre $10 \%$ y $20 \%$, y su rol como factor pronóstico aún está en estudio ${ }^{31}$. No obstante, de algunos resultados contradictorios ${ }^{32}$, la mayor parte de los trabajos recientes apoyan el rol que tiene la sobreexpresión y la amplificación del HER2 en la evolución del cáncer gástrico, así como la utilidad clínica de su determinación para la indicación de la terapia con anticuerpos monoclonales contra el receptor ${ }^{33-35}$. Además, la sobreexpresión de HER2 se ha asociado a enfermedad avanzada, lo que pudiese darle un valor pronóstico a la determinación HER2 en los tejidos tumorales ${ }^{27,36}$.

El estatus de HER2 se determina en los tejidos tumorales, la sobreexpresión proteica mediante técnicas de inmunohistoquímica y la amplificación del gen a través de la visualización directa del número de copias mediante técnicas de marcación fluorescente (FISH), y más recientemente con técnicas cromogénicas (ISH) ${ }^{37-40}$.

De la experiencia obtenida en el cáncer de mama, se han extrapolado algunas pautas para la interpretación de estas determinaciones, restringiéndose la positividad inmunohistoquímica a aquellos casos considerados como francamente positivos $(3+)$, o bien con técnicas de hibridación a los casos con índices de sobre 2,2 señales de HER2 por señal centrómerica del cromosoma $17^{41,42}$. Sin embargo, de estas pautas, los criterios de positividad para el cáncer gástrico presentan un mayor grado de ambigüedad y de dificultad en su interpretación, en comparación a los establecidos para el cáncer de mama ${ }^{43}$. La heterogeneidad en la sobreexpresión y amplificación de HER2 es un problema aún no resuelto. La correlación entre técnicas de inmunohistoquímica y FISH se han reportado en rangos que fluctúan entre 87 y $93 \%$. Nuevas técnicas de análisis de imagen han intentado disminuir las variaciones interpersonales en la interpretación y cuantificación tanto de la tinción inmunohistoquímica como de la medición de señales fluorescentes ${ }^{44}$.

En nuestro país se realizan determinaciones rutinarias de HER2 mediante técnicas de inmunohistoquímica y FISH para el cáncer de mama, sin embargo, no existe información publicada en cáncer gástrico. El objetivo de este trabajo es determinar la frecuencia y distribución de la sobreexpresión inmunohistoquímica y amplificación del gen HER2 en el cáncer gástrico avanzado.

\section{Material y Método}

\section{Casos}

Se incluyen en este estudio 109 pacientes con adenocarcinomas gástricos primarios en estadios avanzados con presencia de metástasis ganglionares de los Hospitales de Puerto Montt, Hospital Padre Hurtado. Hospital Militar y Clínica Alemana de Santiago y Temuco. De cada uno de los centros se seleccionó una inclusión representativa del tumor (material de archivo de tejidos fijados en formalina e incluidos en parafina). Se realizó estudio inmunohistoquímico contra la proteína del gen HER2 y para los casos que mostraron positividad $(2+)$ y $(3+)$ (ver más adelante), se realizó estudio de hibridación fluorescente in situ en el Servicio 
Amplificación de HER2 en cáncer gástrico - I. Roa et al

de Anatomía Patológica de Clínica Alemana de Santiago. Se confeccionó una base de datos anónima de las muestras, en la cual fueron consignados exclusivamente los siguientes parámetros: género, edad, tipo histológico y localización del tumor. Se reconocieron tres tipos histológicos ${ }^{45}$ : intestinal, difuso de acuerdo a las definiciones establecidas y un tercer grupo o mixto, en el cual se observan las características de ambos tipos histológicos.

\section{Confección de matrices de tejidos (TMA)}

De cada una de las muestras se seleccionaron 3 áreas del tumor, las que fueron señalizadas en los cortes teñidos con HE con un objetivo marcador Nikon. Para la confección de las matrices de tejidos se utilizó el Semiautomatic Tissue MicroArrayer Pathology Devices, Inc con agujas de $2 \mathrm{~mm}$ de diámetro. Los cilindros de tejido fueron extraídos del bloque donante y puestos en el bloque receptor. Las inclusiones fueron puestas en estufa a $55^{\circ} \mathrm{C}$ por 30 min para obtener la adherencia entre las muestras y el bloque receptor.

\section{Técnica de inmunohistoquímica}

Se realizó la técnica estándar para tejidos fijados en formalina e incluidos en parafina. Los cortes histológicos de $4 \mu \mathrm{m}$ de espesor obtenidos de los TMA fueron desparafinados e hidratados en concentraciones decrecientes de alcohol. La recuperación de antígenos se realizó mediante exposición a microondas en buffer citrato $\mathrm{pH}$ 6,0 y lavados en PBS pH 7.4. Se utilizó el anticuerpo monoclonal c-erb-B2 (NCL-356) Novocastra en una dilución de $1 / 40$. El anticuerpo primario fue incubado a temperatura ambiente por $60 \mathrm{~min}$ y luego incubado con el complejo Super Picture Polymer Detection $\mathrm{Kit}^{\mathrm{TM}}$ Zymed en equipo Dakoautostainer ${ }^{\mathrm{TM}}$.

\section{Formas de medición de la positividad}

Se realizó de acuerdo a la recomendación sugerida por Hofmann et al, $2008^{42}$ que se resume en la Tabla 1 . Se consideró como positivo a la tinción de membrana lateral o basolateral completa con intensidad $(3+)$ en un porcentaje de al menos

\section{Tabla 1. Se describe el método de cuantificación de la positividad inmunohistoquímica (sobreexpresión) e hibridación in situ (amplificación)}

\begin{tabular}{|c|c|c|c|}
\hline & \multicolumn{3}{|c|}{ Medición de la sobreexpresión y amplificación de HER2 } \\
\hline \multicolumn{4}{|c|}{ Inmunohistoquímica } \\
\hline & Muestras quirúrgicas & Muestras endoscópicas & \\
\hline $\begin{array}{l}\text { Intensidad } \\
\text { de tinción }\end{array}$ & Patrón de tinción & Patrón de tinción & $\begin{array}{l}\text { Sobreexpresión } \\
\text { HER2 }\end{array}$ \\
\hline 0 & $\begin{array}{l}\text { No se observa tinción o la tinción de } \\
\text { membrana se observa en }<10 \% \text { de las } \\
\text { células tumorales }\end{array}$ & $\begin{array}{l}\text { No se observa tinción en ninguna célula } \\
\text { tumoral }\end{array}$ & Negativo \\
\hline $1+$ & $\begin{array}{l}\text { Leve y perceptible tinción de membra- } \\
\text { na incompleta }>10 \% \text { de las células } \\
\text { tumorales }\end{array}$ & $\begin{array}{l}\text { Leve y perceptible tinción de membrana } \\
\text { de grupos de } 5 \text { o más células tumorales } \\
\text { (cualquier \%) }\end{array}$ & Negativo \\
\hline $2+$ & $\begin{array}{l}\text { Tinción débil a moderada completa de } \\
\text { membrana lateral o basolateral en }>10 \\
\% \text { de las células tumorales }\end{array}$ & $\begin{array}{l}\text { Tinción leve o moderada completa de } \\
\text { membrana lateral o basolateral (cual- } \\
\text { quier \%) }\end{array}$ & Equívoca \\
\hline $3+$ & $\begin{array}{l}\text { Tinción intensa completa de membra- } \\
\text { na lateral o basolateral >10 \% de las } \\
\text { células tumorales }\end{array}$ & $\begin{array}{l}\text { Tinción intensa completa de membrana } \\
\text { lateral o basolateral (cualquier \%) }\end{array}$ & Positiva \\
\hline \multicolumn{4}{|l|}{ FISH } \\
\hline $\begin{array}{l}\text { Relación } \\
\text { HER2: C17P }\end{array}$ & Interpretación & Criterio & \\
\hline$<2,0$ & Amplificación negativa & $\begin{array}{l}\text { Conteo de a lo menos } 20 \text { núcleos } \\
\text { tumorales }\end{array}$ & \\
\hline$>2,0$ & Amplificación positiva & $\begin{array}{l}\text { Se recomienda contar más núcleos si la } \\
\text { relación está entre } 1,8-2,2\end{array}$ & \\
\hline
\end{tabular}




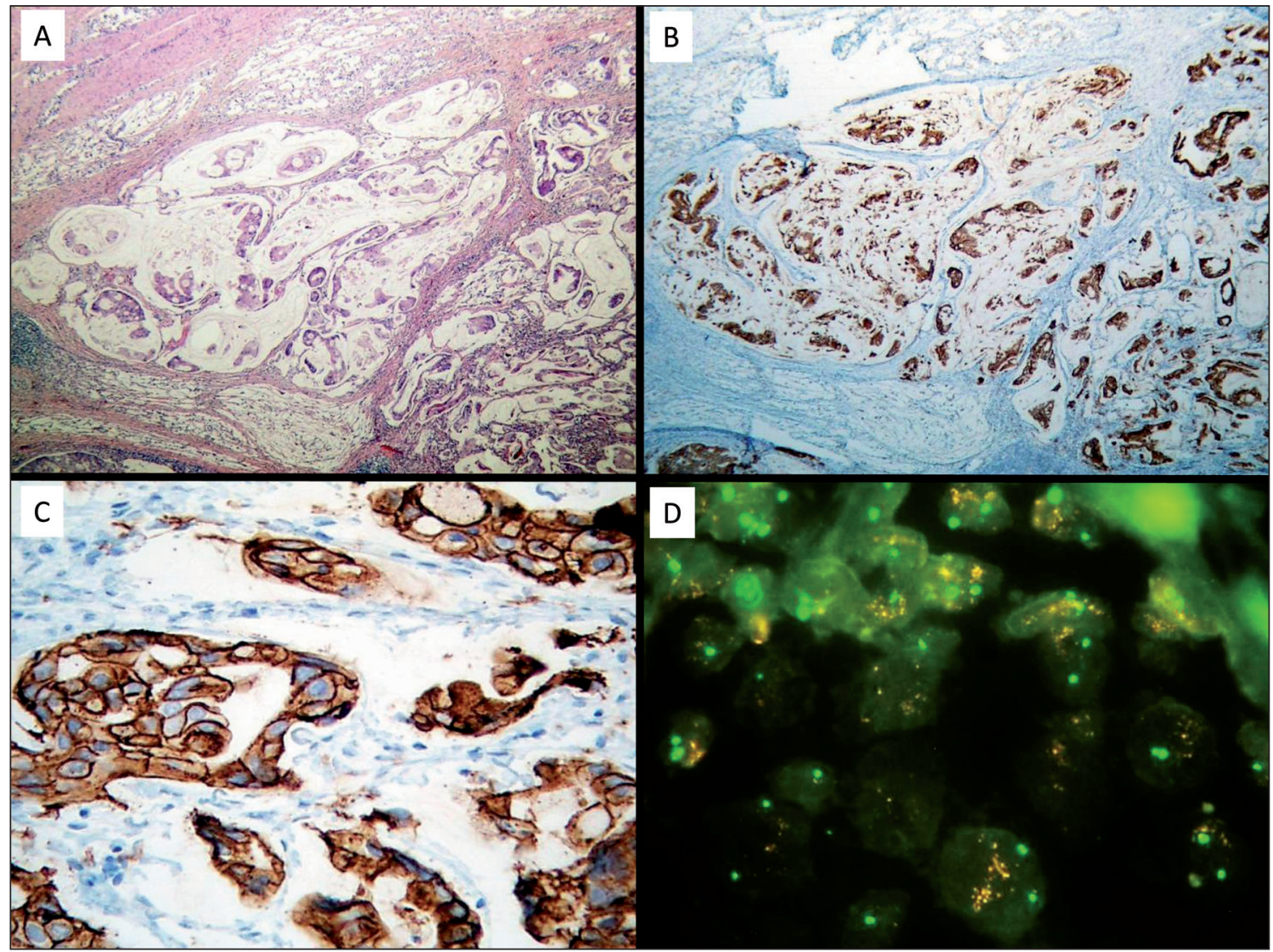

Figura 1. A: Adenocarcinoma gástrico de tipo intestinal con signos de mucosecreción con formación de lagos de mucina y brotes epiteliales tumorales (HE X 32). B: Tinción inmunohistoquímica que se logra distinguir en la mayoría de los grupos tumorales a bajo aumento (HE X 32). C: Magnificación de la tinción positiva, intensa lateral y basolateral en la mayoría de las células tumorales (3+). D: Hibridación in situ fluorescente con dos señales verdes correspondientes a la marcación centromérica del cromosoma 17 y múltiples señales anaranjadas intranucleares con una relación HER2/C17 > 2 (X 1000).

$10 \%$ de las células tumorales (Figura 1). Los casos considerados como equívocos $(2+)$ y positivos fueron procesados para hibridación in situ.

\section{Técnica de hibridación in situ}

Se realizó en cortes de $4 \mu \mathrm{m}$ obtenidos de los TMA. En los casos con discrepancia entre la inmunohistoquímica e hibridación in situ, se repitió la hibridación en la muestra completa, a fin de disminuir la posibilidad de error por una inadecuada localización del área positiva. Resumidamente la técnica consistió en desparafinización de las muestras en xilol e hidratación en concentraciones decrecientes de alcohol. La hibridación se realizó de acuerdo a las especificaciones del fabricante con el método Dako Cytomation con Kit HER2 FISH
pharmDx ${ }^{\mathrm{TM}}$ en Hibridador DAKO en tiempos $\mathrm{y}$ temperaturas de acuerdo a las especificaciones del fabricante. Se utilizó como control positivo muestras de cánceres de mama con HER2 $(3+)$ y FISH positivo con un índice mayor de 6 señales.

\section{Formas de medición de la positividad}

Se realizó de acuerdo a pautas sugeridas con conteo simultáneo de señales en 30 núcleos tumorales aislados e intactos. Ambas señales fueron capturadas en las longitudes de onda específicas para fluorescencia verde y roja en cámara Olympus DP72 y procesamiento de imagen con el programa cellSens. En imágenes integradas se realizó el conteo de señales Cen17 y de HER2 (Figura 1). Se consideró como amplificación del gen HER2 
Amplificación de HER2 en cáncer gástrico - I. Roa et al

cuando el cociente del número de señales HER2/ Cen17 fue superior a 2,2 (Foto 2), negativo inferior a 1,8 y como no concluyente entre 1,8 y $2,2^{37}$. En estos casos las preparaciones fueron reexaminadas con un nuevo conteo de 30 núcleos más.

\section{Análisis estadístico}

Se realizó mediante test de $\chi^{2}$ y exacto de Fisher para las tablas de contingencia $(\mathrm{p}<0,05)$.

\section{Resultados}

El grupo total estaba constituido por 109 casos, 33 casos (30\%) correspondieron a mujeres con un promedio de edad de 61,9 años (DS $\pm 13,8$ años) y los restantes 76 casos a hombres con un promedio de edad de 66,6 años (DS $\pm 11,6$ años). La totalidad de los casos correspondieron a adenocarcinomas. Los tumores primarios se localizaron en el cuerpo en 49 casos $(44,9 \%), 37$ casos en el antro $(36,6 \%)$, 15 casos en el cardias $(13,7 \%)$ y en el fondo en 4 casos $(3,7 \%)$. No se logró precisar la localización del tumor en 4 casos $(3,7 \%)$. En 61 casos $(56 \%)$ los tumores eran de tipo intestinal y en 19 casos $(17,4 \%)$ de tipo difuso. Los 29 casos restantes $(26,6 \%)$ eran tumores de tipo mixto con áreas de tipo intestinal y difuso (Tabla 2). En las mujeres los tres tipos histológicos se distribuyeron homogéneamente, en cambio en los hombres predominaron los de tipo intestinal $(63,2 \%)$ y sólo $11,8 \%$ (9 casos) fueron de tipo difuso. La diferencia en la distribución de tipos histológicos por género fue significativa $(\mathrm{p}=0,03)$.

La tinción inmunohistoquímica mostró positividad intensa (3+) en 11 casos $(10,9 \%)$ y fue negativa en $54 \%$ de los casos ( 43 casos sin tinción y 16 casos $(1+))$; los restantes 39 casos fueron equívocos o tinción $(2+)$. De los 11 casos positivos $(3+)$, sólo 2 eran mujeres (6,1\%), en cambio, 9 de 76 hombres fueron positivos $(15,8 \%)(\mathrm{p}=\mathrm{ns}) . \mathrm{La}$ expresión inmunohistoquímica y su relación con los distintos tipos histológicos se muestran en la Tabla 3. Es destacable que los casos positivos (3+) se observaron en 10 de los 11 tumores de tipo intestinal, es decir, 16,4\% del total de tumores de

Tabla 2. Distribución de los tipos histológicos en relación al género

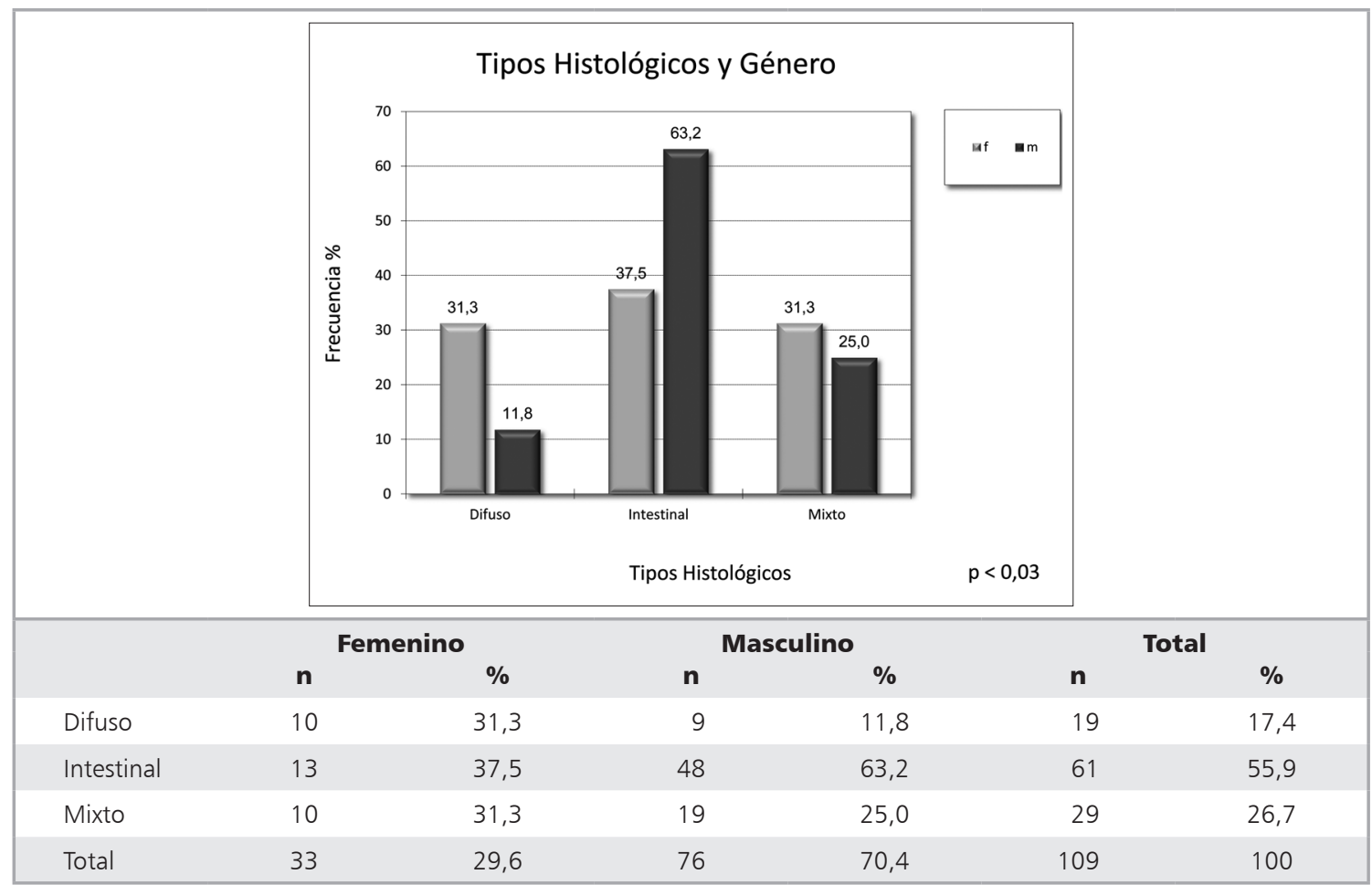


Tabla 3. Expresión inmunohistoquímica de HER2 en relación a los tipos histológicos

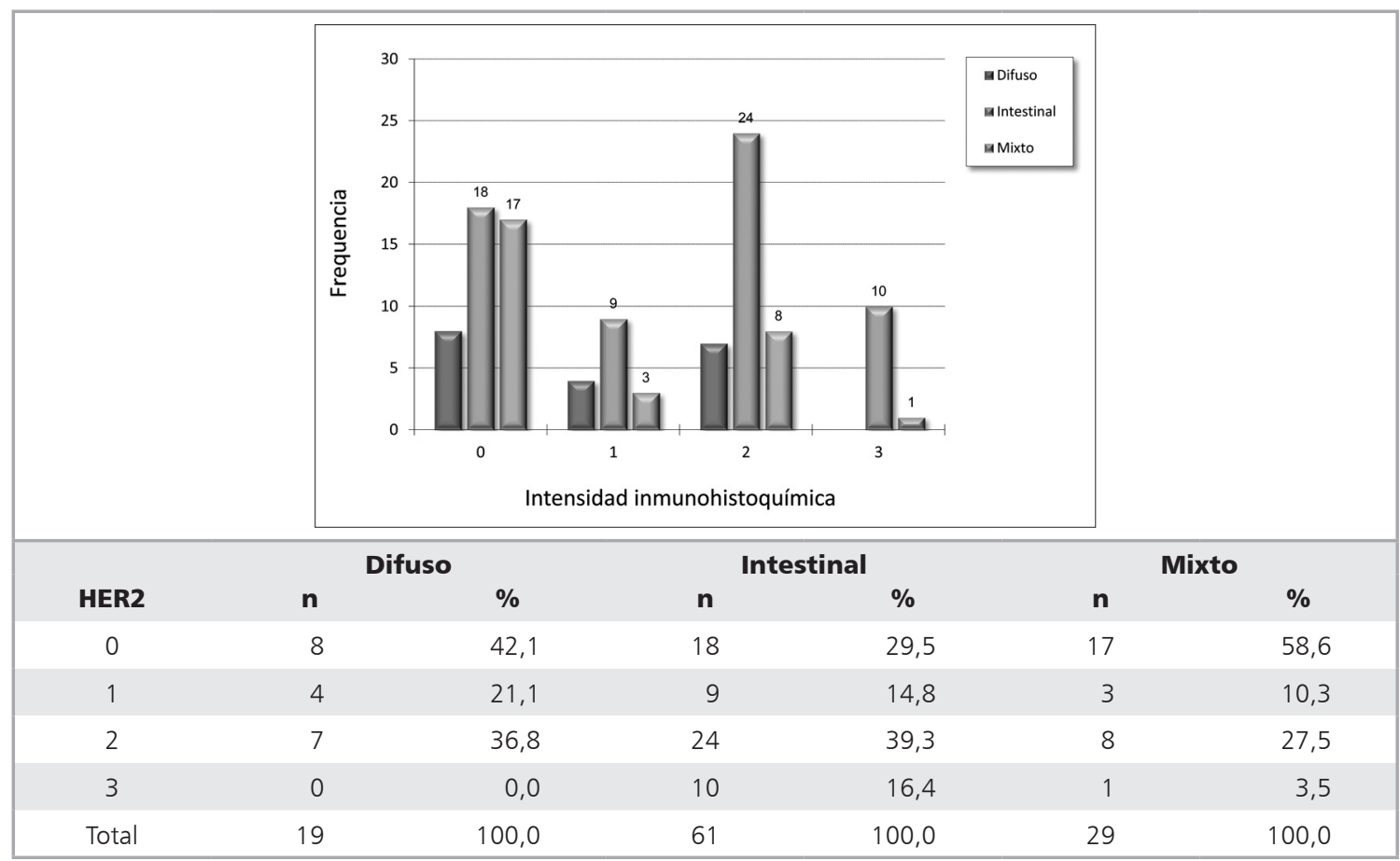

tipo intestinal. El caso positivo restante fue de tipo mixto (3,5\%), cuya positividad fue observada sólo en el área con diferenciación tubular. Ninguno de los tumores difusos mostró positividad para HER2. En tres casos se observó positividad inmunohistoquímica $(3+)$, pero en porcentajes que fluctuaron entre 0,29\% y 2,1\% del área total del tumor. En estos tres casos se realizó hibridación in situ dirigida al área de positividad, resultando los tres casos negativos.

La hibridación in situ se realizó en 50 casos correspondientes a 39 casos con intensidad inmunohistoquímica $(2+)$ y a los 11 casos $(3+)$. Los 11 casos (3+) fueron positivos en la hibridación y dos casos con tinción $(2+)$. Es decir, en 2 de 39 casos $(2+)(5,7 \%)$, se logró demostrar amplificación de aquellos casos considerados como equívocos siendo el restante $94,3 \%$ negativos. Dos casos fueron considerados como no concluyentes a pesar del recuento de un mayor número de núcleos tumorales y un caso fue considerado como inadecuado para estudio.

La concordancia global observada entre la tinción inmunohistoquímica y la hibridación in situ alcanzó a 85\%, detectándose más casos positivos con la hibridación in situ que con la inmunohistoquímica a expensas del grupo de los equívocos o $(2+)$, lo cual muestra consistencia entre ambas determinaciones. El escaso número de observaciones y la negatividad de algunas variables no permitieron realizar otros análisis estadísticos.

\section{Discusión}

Nuestros resultados muestran sobreexpresión y/o amplificación del gen HER2 en 11,9\% de las muestras de cánceres gástricos avanzados operados. La diferencia observada entre la inmunohistoquímica y la hibridación in situ fue consecuencia del estudio de todos los casos considerados como equívocos o $(2+)$, que en esta serie correspondieron a 39 casos $(35,7 \%)$, grupo en el cual se detectaron dos casos amplificados, lo cual traduce la baja frecuencia de amplificación en este grupo de pacientes. Considerando el alto costo que tiene el examen de hibridación in situ, sumado a esto el alto consumo de tiempo de observación 
por ser láminas completas, en las que se requiere examinar múltiples campos, se debería evaluar su justificación y utilidad, ya que casi 95\% de estas determinaciones resultan negativas.

Tal y como está reportado en series extranjeras la sobreexpresión/amplificación de HER2, se observa con significativa mayor frecuencia en los tumores mejor diferenciados o de tipo intestinal ${ }^{34}$. En nuestros casos esta positividad alcanzó a 16,4\%. Por su parte, los tumores con una histología bifásica (histológicamente mixtos) mostraron muy baja positividad $(3,5 \%)$ y los de tipo difuso fueron todos negativos. Este hecho sugiere que la determinación del estatus HER2, debería reservarse para aquellos pacientes portadores tumores de tipo intestinal ${ }^{46}$. Es importante también señalar que la heterogeneidad observada tanto en la tinción inmunohistoquímica como en la amplificación del gen HER2 en el cáncer gástrico lo hace diferente a otras neoplasias como el cáncer de mama, lo cual restringe la validez de sus resultados ${ }^{47-49}$. Estas marcadas diferencias intratumorales es una de las mayores limitaciones del método que determina su poca aplicabilidad a biopsias endoscópicas, las cuales podrían mostrar resultados negativos producto exclusivamente del muestreo ${ }^{50}$. Como señalamos en tres de nuestros casos, a pesar de tener áreas intensamente positivas $(3+)$ dentro del tumor, si hubiesen sido detectadas en biopsias endoscópicas, estos casos habrían sido considerados como positivos, sin embargo, no cumplían los criterios de positividad establecidos para las biopsias quirúrgicas ${ }^{42}$. Por otro lado, la negatividad de biopsias endoscópicas, tampoco garantiza que el tumor sea negativo dadas las mismas consideraciones anteriores.

Finalmente, podemos concluir que la determinación de la sobreexpresión de HER2 en el cáncer gástrico avanzado en nuestro medio, representa un pequeño avance a pesar de beneficiar sólo a un selecto grupo de pacientes, pero abre expectativas a la determinación de otros genes, así como el uso de terapia dirigida a estos blancos moleculares en esta tan frecuente enfermedad maligna.

\section{Referencias}

1. Stomach Cancer Incidence and Mortality Worldwide in 2008. Summary. http://globocan.iarc.fr/factsheets/ cancers/stomach.asp 2008.

2. Guggenheim DE, Shah MA. Gastric cancer epidemiology and risk factors. J Surg Oncol 2013; 107 (3): 230-6.

3. Crew KD, Neugut AI. Epidemiology of gastric cancer. World J Gastroenterol 2006; 12 (3): 354-62.

4. Bosetti C, Malvezzi M, Chatenoud L, Negri E, Levi F, La Vecchia C. Trends in cancer mortality in the Americas, 1970-2000. Ann Oncol 2005; 16 (3): 489-511.

5. Tapia E O, Villaseca H M, Araya O JC, Roa S JC. [Prognostic value of extracapsular lymph node involvement among patients with gastric cancer]. Rev Med Chile 2011; 139 (8): 992-7.

6. Torres J, Correa P, Ferreccio C, Hernández-Suárez G, Herrero R, Cavazza-Porro M, et al. Gastric cancer incidence and mortality is associated with altitude in the mountainous regions of Pacific Latin America. Cancer Causes Control 2013; 24 (2): 249-56.

7. Bertuccio P, Chatenoud L, Levi F, Praud D, Ferlay J, Negri E, et al. Recent patterns in gastric cancer: a global overview. Int J Cancer 2009; 125 (3): 666-73.

8. Shridhar R, Almhanna K, Hoffe SE, Fulp W, Weber J, Chuong $\mathrm{MD}$, et al. Increased survival associated with surgery and radiation therapy in metastatic gastric cancer: A Surveillance, Epidemiology, and End Results database analysis 10.1002/cncr.27927. Cancer 2013.

9. Morabito A, Carillio G, Longo R. Systemic treatment of gastric cancer. Crit Rev Oncol Hematol 2009; 70 (3): 216-34.

10. García CC, Benavides CC, Apablaza SP, Rubilar PO, Covacevich SR, Peñaloza PM, et al. [Surgical treatment of gastric cancer: results in 423 cases]. Rev Med Chile 2007; 135 (6): 687-95.

11. Butte JM, Torres J, Viviani P, Duarte I, Crovari F, Guzmán S, et al. [Long term survival of patients operated for early gastric cancer]. Rev Med Chile 2008; 136 (11): 1424-30.

12. Wong H, Yau T. Targeted therapy in the management of advanced gastric cancer: are we making progress in the era of personalized medicine? Oncologist 2012; 17 (3): 346-58.

13. Terashima M, Kitada K, Ochiai A, Ichikawa W, Kurahashi I, Sakuramoto S, et al. Impact of expression of human epidermal growth factor receptors EGFR and ERBB2 on survival in stage II/III gastric cancer. Clin Cancer Res 2012; 18 (21): 5992-6000.

14. Chua TC, Merrett ND. Clinicopathologic factors associated with HER2-positive gastric cancer and its impact on survival outcomes-a systematic review. Int J Cancer 2012; 130 (12): 2845-56.

15. Stern HM. Improving treatment of HER2-positive cancers: opportunities and challenges. Sci Transl Med 2012; 4 (127): 127rv2.

16. English DP, Roque DM, Santin AD. HER2 Expression 
Beyond Breast Cancer: Therapeutic Implications for Gynecologic Malignancies. Mol Diagn Ther 2013; 17 (2): 85-99.

17. Lauren P. The Two Histological Main Types of Gastric Carcinoma: Diffuse and So-Called Intestinal-Type Carcinoma. An Attempt at a Histo-Clinical Classification. Acta Pathol Microbiol Scand 1965; 64: 31-49.

18. Hu B, El Hajj N, Sittler S, Lammert N, Barnes R, MeloniEhrig A. Gastric cancer: Classification, histology and application of molecular pathology. J Gastrointest Oncol 2012; 3 (3): 251-61.

19. Deng N, Goh LK, Wang H, Das K, Tao J, Tan IB, et al. A comprehensive survey of genomic alterations in gastric cancer reveals systematic patterns of molecular exclusivity and co-occurrence among distinct therapeutic targets. Gut 2012; 61 (5): 673-84.

20. Carneiro F, Oliveira C, Leite M, Seruca R. Molecular targets and biological modifiers in gastric cancer. Semin Diagn Pathol 2008; 25 (4): 274-87.

21. Yamashita K, Sakuramoto S, Watanabe M. Genomic and epigenetic profiles of gastric cancer: potential diagnostic and therapeutic applications. Surg Today 2011; 41 (1): 24-38.

22. Lynch HT, Grady W, Suriano G, Huntsman D. Gastric cancer: new genetic developments. J Surg Oncol 2005; 90 (3): 114-33; discussion 133.

23. Liu YC, Shen CY, Wu HS, Hsieh TY, Chan DC, Chen CJ, et al. Mechanisms inactivating the gene for E-cadherin in sporadic gastric carcinomas. World J Gastroenterol 2006; 12 (14): 2168-73.

24. Oliveira C, Sousa S, Pinheiro H, Karam R, BordeiraCarrico R, Senz J, et al. Quantification of epigenetic and genetic 2nd hits in $\mathrm{CDH} 1$ during hereditary diffuse gastric cancer syndrome progression. Gastroenterology 2009; 136 (7): 2137-48.

25. Tai W, Mahato R, Cheng K. The role of HER2 in cancer therapy and targeted drug delivery. J Control Release 2010; 146 (3): 264-75.

26. Rexer BN, Arteaga CL. Intrinsic and acquired resistance to HER2-targeted therapies in HER2 gene-amplified breast cancer: mechanisms and clinical implications. Crit Rev Oncog 2012; 17 (1): 1-16.

27. Bayrak M, Olmez OF, Kurt E, Cubukcu E, Evrensel T, Kanat O, et al. Prognostic significance of c-erbB2 overexpression in patients with metastatic gastric cancer. Clin Transl Oncol 2013; 15 (4): 307-12.

28. Bang YJ, Van Cutsem E, Feyereislova A, Chung HC, Shen L, Sawaki A, et al. Trastuzumab in combination with chemotherapy versus chemotherapy alone for treatment of HER2-positive advanced gastric or gastro-oesophageal junction cancer (ToGA): a phase 3, open-label, randomised controlled trial. Lancet 2010; 376 (9742): 687-97.

29. Okines AF, Cunningham D. Trastuzumab in gastric cancer. Eur J Cancer 2010; 46 (11): 1949-59.

30. Chen CT, Kim H, Liska D, Gao S, Christensen JG, Weiser MR. MET activation mediates resistance to lapatinib inhibition of HER2-amplified gastric cancer cells. Mol Cancer Ther 2012; 11 (3): 660-9.

31. Hechtman JF, Polydorides AD. HER2/neu gene amplification and protein overexpression in gastric and gastroesophageal junction adenocarcinoma: a review of histopathology, diagnostic testing, and clinical implications. Arch Pathol Lab Med 2012; 136 (6): 691-7.

32. Halon A, Donizy P, Biecek P, Rudno-Rudzinska J, Kielan W, Matkowski R. HER-2 expression in immunohistochemistry has no prognostic significance in gastric cancer patients. Scientific World Journal 2012; 2012: 941259.

33. Smyth EC, Cunningham D. Targeted therapy for gastric cancer. Curr Treat Options Oncol 2012; 13 (3): 377-89.

34. Liu W, Zhong S, Chen J, Yu Y. HER-2/neu overexpression is an independent prognostic factor for intestinal-type and early-stage gastric cancer patients. J Clin Gastroenterol 2012; 46 (4): 31-7.

35. Kim JW, Im SA, Kim M, Cha Y, Lee KH, Keam B, et al. The prognostic significance of HER2 positivity for advanced gastric cancer patients undergoing first-line modified FOLFOX-6 regimen. Anticancer Res 2012; 32 (4): 1547-53.

36. Yu GZ, Chen Y, Wang JJ. Overexpression of Grb2/HER2 signaling in Chinese gastric cancer: their relationship with clinicopathological parameters and prognostic significance. J Cancer Res Clin Oncol 2009; 135 (10): 1331-9.

37. Räschoff J, Hanna W, Bilous M, Hofmann M, Osamura RY, Penault-Llorca F, et al. HER2 testing in gastric cancer: a practical approach. Mod Pathol 2012; 25 (5): 637-50.

38. Park YS, Hwang HS, Park HJ, Ryu MH, Chang HM, Yook JH, et al. Comprehensive analysis of HER2 expression and gene amplification in gastric cancers using immunohistochemistry and in situ hybridization: which scoring system should we use? Hum Pathol 2012; 43 (3): 413-22.

39. Hirschmann A, Lamb TA, Marchal G, Padilla M, Diebold J. Simultaneous analysis of HER2 gene and protein on a single slide facilitates HER2 testing of breast and gastric carcinomas. Am J Clin Pathol 2012; 138 (6): 837-44.

40. Cho EY, Srivastava A, Park K, Kim J, Lee MH, Do I, et al. Comparison of four immumohistochemical tests and FISH for measuring HER2 expression in gastric carcinomas. Pathology 2012; 44 (3): 216-20. 
Amplificación de HER2 en cáncer gástrico - I. Roa et al

41. Mrklic I, Bendic A, Kunac N, Bezic J, Forempoher G, Durdov MG, et al. Her-2/neu assessment for gastric carcinoma: validation of scoring system. Hepatogastroenterology 2012; 59 (113): 300-3.

42. Hofmann M, Stoss O, Shi D, BÃ $1 / 4$ ttner R, van de Vijver M, Kim W, et al. Assessment of a HER2 scoring system for gastric cancer: results from a validation study. Histopathology 2008; 52 (7): 797-805.

43. Stenzinger A, von Winterfeld M, Aulmann S, Warth A, Weichert W, Denkert C, et al. Quantitative analysis of diagnostic guidelines for HER2-status assessment. J Mol Diagn 2012; 14 (3): 199-205.

44. Jeung J, Patel R, Vila L, Wakefield D, Liu C. Quantitation of HER2/neu expression in primary gastroesophageal adenocarcinomas using conventional light microscopy and quantitative image analysis. Arch Pathol Lab Med 2012; 136 (6): 610-7.

45. WHO Classification of Tumours of the Digestive System, Fourth Edition. 2010. IARC Press, Lyon.

46. Penault-Llorca F, Chenard MP, Bouchon, Emile JF, Bibeau F, Metges JP, et al. [HER2 and gastric cancer. Re- commendations for clinical practice in 2011. Ann Pathol 2011; 31 (2): 78-87.

47. Yoon Cho E, Park K, Do I, Cho J, Kim J, Lee J, et al. Heterogeneity of ERBB2 in gastric carcinomas: a study of tissue microarray and matched primary and metastatic carcinomas. Mod Pathol 2012.

48. Yang J, Luo H, Li Y, Li J, Cai Z, Su X, et al. Intratumoral heterogeneity determines discordant results of diagnostic tests for human epidermal growth factor receptor (HER) 2 in gastric cancer specimens. Cell Biochem Biophys 2012; 62 (1): 221-8.

49. Grob TJ, Kannengiesser I, Tsourlakis MC, Atanackovic D, Koenig AM, Vashist YK, et al. Heterogeneity of ERBB2 amplification in adenocarcinoma, squamous cell carcinoma and large cell undifferentiated carcinoma of the lung. Modern Pathol 2012; 25 (12): 1566-73.

50. Asioli S, Maletta F, Verdun di Cantogno L, Satolli MA, Schena M, Pecchioni C, et al. Approaching heterogeneity of human epidermal growth factor receptor 2 in surgical specimens of gastric cancer. Hum Pathol 2012; 43 (11): 2070-9. 